\title{
Correction to: Contribution of Zinc-Dependent Delayed Calcium Influx via TRPC5 in Oxidative Neuronal Death and its Prevention by Novel TRPC Antagonist
}

\begin{abstract}
Sang Eun Park ${ }^{1}$ - Ji Hoon Song ${ }^{1}$ - Chansik Hong ${ }^{2}$ • Dong Eun Kim ${ }^{1}$ - Jee-Won Sul ${ }^{1}$ Tae-Youn Kim ${ }^{1,3}$ - Bo-Ra Seo ${ }^{3}$. Insuk So ${ }^{4}$. Sang-Yeob Kim ${ }^{1,5}$. Dong-Jun Bae ${ }^{1}$ - Mi-Ha Park ${ }^{1}$ • Hye Min Lim ${ }^{1}$ • In-Jeoung Baek ${ }^{1,5}$. Antonio Riccio ${ }^{6,7}$. Joo-Yong Lee ${ }^{1,5}$. Woo Hyun Shim ${ }^{1,5} \cdot$ Bumwoo Park ${ }^{1,8} \cdot$ Jae-Young Koh ${ }^{3,9} \cdot$ Jung Jin Hwang ${ }^{1,5}$ (D)
\end{abstract}

Published online: 12 December 2018

(C) Springer Science+Business Media, LLC, part of Springer Nature 2018

\section{Correction to: Mol Neurobiol} https://doi.org/10.1007/s12035-018-1258-7

After the publication of this work errors were noticed in Fig. $3 \mathrm{~b}$ and $4 \mathrm{~d}$.

Figure 3b) Image of TPEN is originally image of NU. I missed out the image of TPEN. This confusion is judged to be a mistake during data processing.

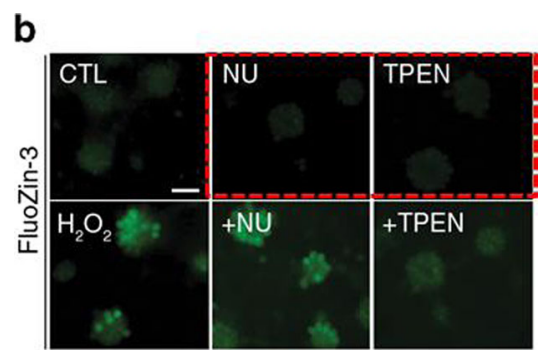

The online version of the original article can be found at https://doi.org/ $10.1007 / \mathrm{s} 12035-018-1258-7$

Jae-Young Koh

jkko@amc.seoul.kr

$\triangle$ Jung Jin Hwang

jjhwang@amc.seoul.kr

1 Asan Institute for Life Sciences, Asan Medical Center, Seoul 05505, South Korea

2 Department of Physiology, Chosun University School of Medicine, Kwangju 61452, South Korea

3 Neural Injury Research Lab, University of Ulsan College of Medicine, Seoul 05505, South Korea

4 Department of Physiology and Institute of Dermatological Science, Seoul National University College of Medicine, Seoul 110-799, South Korea
Therefore, I change image of NU and TPEN to correct one as below.

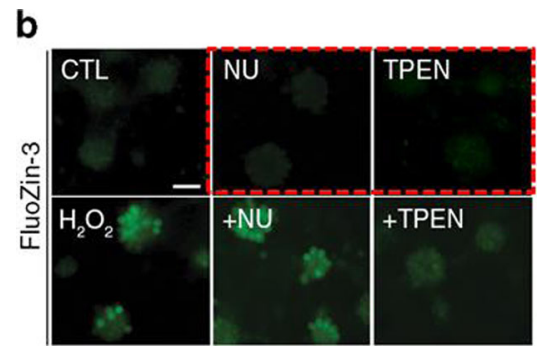

Department of Convergence Medicine, University of Ulsan College of Medicine, 88, Olympic-ro 43-gil, Songpa-Gu, Seoul 05505, South Korea

6 Department of Cardiology, Boston Children's Hospital, Boston, MA, USA

Department of Neurobiology, Harvard Medical School, Boston, MA, USA

8 Biomedical Engineering, Ulsan National Institute of Science and Technology, Ulsan 44919, South Korea

9 Department of Neurology, Asan Medical Center, University of Ulsan College of Medicine, 88, Olympic-ro 43-gil, Songpa-Gu, Seoul 05505, South Korea 
Figure 4d) Images of ML204 alone and H2O2 + ML204 were accidentally duplicated.

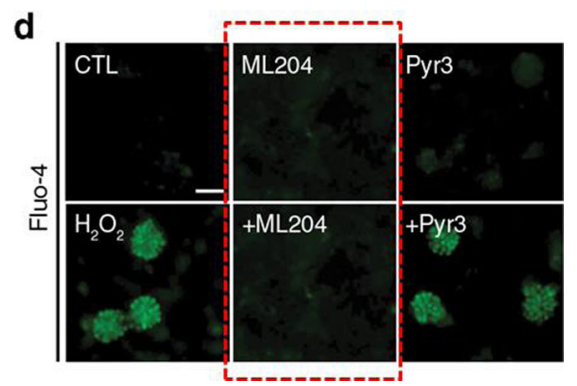

Therefore, I change the image of ML204 alone to correct one as below.

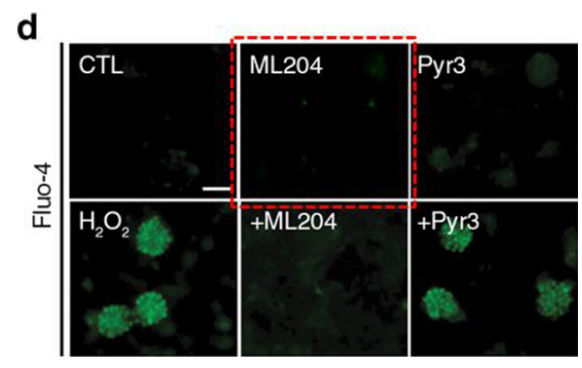

Publisher's Note Springer Nature remains neutral with regard to jurisdictional claims in published maps and institutional affiliations. 